\title{
In-situ TEM Investigation of the Amorphous to Crystalline Phase Change During Electrical Breakdown of Highly Conductive Polymers at the Atomic Scale
}

\author{
Michele Conroy, Kalani Moore, Rob Lehane, Alonso Gamero-Quijano, Micheal Scanlon and Ursel \\ Bangert
}

University of Limerick, Limerick, Limerick, Ireland

Flexible electronics has been a field of intense research focus for the diverse and new class of applications not achievable by wafer-based electronics. [1-4] Polymers that are both conductive and stretchable have been put forward as a promising candidate for these device platforms. Due to the often amorphous nature of these material platforms the failure analysis knowledge gained from more traditional devices cannot be applied. The progression and innovation of flexible nanoelectronic manufacturing is dependent on understanding the fundamental physics governing the electronic breakdown of such materials and how to avoid this. In this study we investigate the highly conductive flexible amorphous 2D PEDOT [5-7] layers formed via liquid-liquid interface growth, Figure 1 (a). Utilising aberration corrected TEM and new fast camera technology we study the phase change from amorphous to crystalline at the atomic resolution by in-situ biasing.

The 2D PEDOT layer's structure was initially investigated by transmission electron microscopy (TEM) revealing the finely packed fibrous nature of the amorphous material, Figure 2 (b). Probe measurements of the bulk material revealed surprisingly highly conductive results. Monochromated low loss electron energy loss spectroscopy (EELS) characterisation shows the metallic like conductivity is possibly due to an increase in the density of states, not seen in reduced (less conductive PEDOT). Additionally, the sample itself unlike other polymers was capable of dealing with impressively high dose compared to other polymers/soft materials, possibly due to the ease of high carrier mobility through the material. As shown in the optical microscope images of Figure 1 (a) and (c) the PEDOT layer appears on one side as metallic in colouring. Initially the PEDOT layers biased and monitored under the light microscope for changes in colouring. At electrical breakdown a fine line different in colour formed in the middle of the material between the contacts. To investigate the possible changes structurally during electrical breakdown we utilised the high spatial resolution of TEM using an in-situ biasing DENS holder. We will detail a new scooping sample preparation technique for in-situ biasing of 2D soft materials producing a uniform coating over the contacts as shown in Figure $2(\mathrm{~d})$.

In operando failure analysis at extreme device conditions down to the atomic scale has only recently been made possible by advances in TEM in-situ holder technology and fast cameras. In this study the use of high signal to noise aberration corrected imaging at a high frame rate and low sample drift provided by in-situ TEM MEMS chips made it possible to observe the formation of nucleation clusters and subsequent crystal growth mechanism during electrical breakdown, Figure 2. 4k X 4k TEM imaging at 12 frames per second was used with a ramp rate of $0.001 \mathrm{~V}$ per step to capture the nucleation and crystal growth pathway for the first time of conducting polymer crystal degradation. Several experiments were repeated to investigate the relationship between the density, size and twinning defects of the crystals formed with changing ramp of applied voltage. Fine structure core loss EELS was used to characterise the elemental composition and bonding differences between the particles and the amorphous film and compared to the pre biased sample. The structural evolution of the nanocrystals in terms of position, size and facet formation was then tracked and analysed by post processing software, giving new insights to the phase change dependency on voltage ramp rate. 
(a)

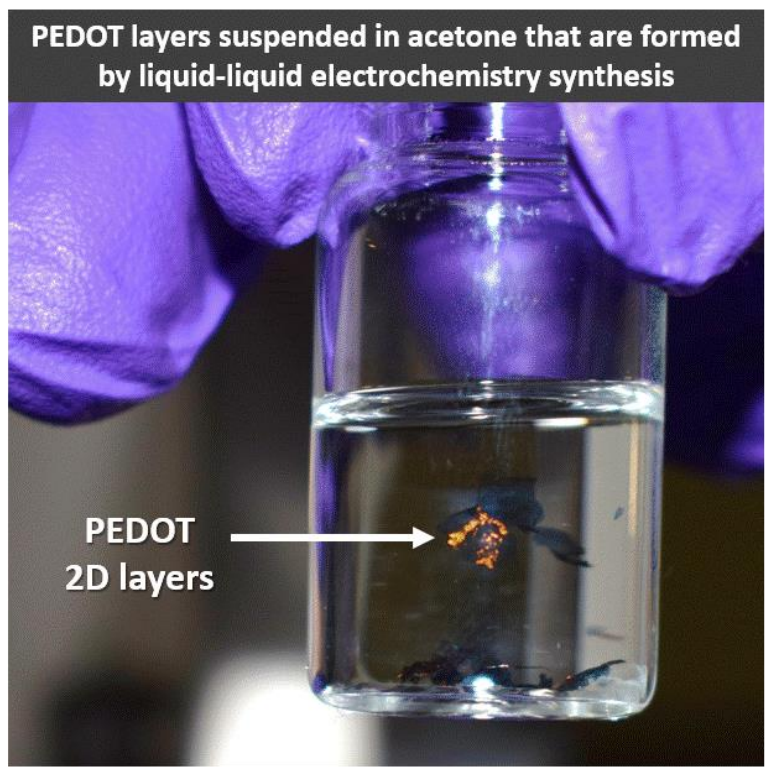

(c)

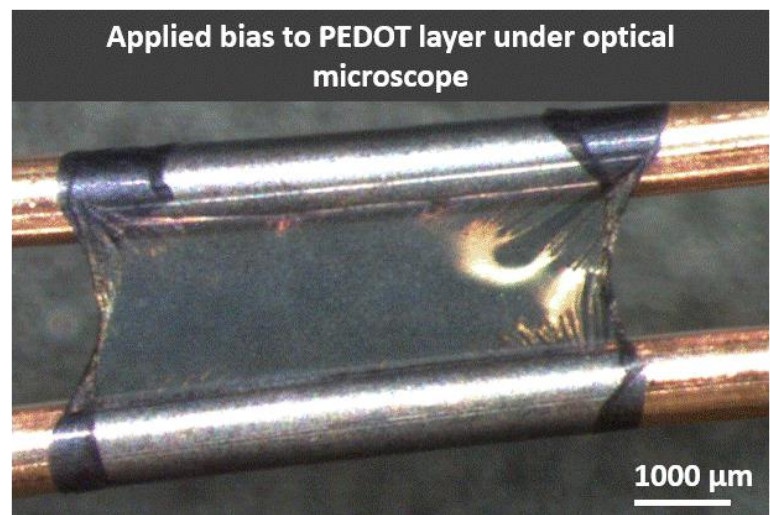

(b)
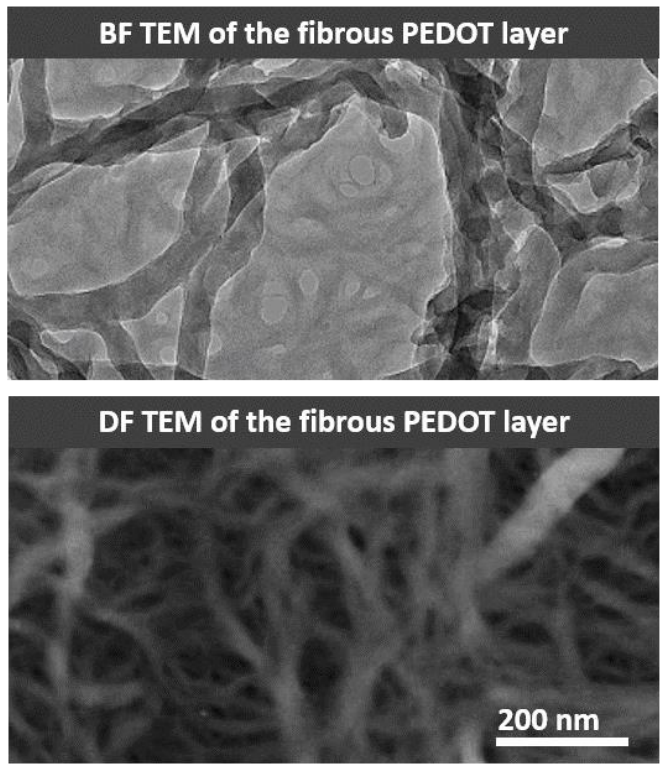

(d)

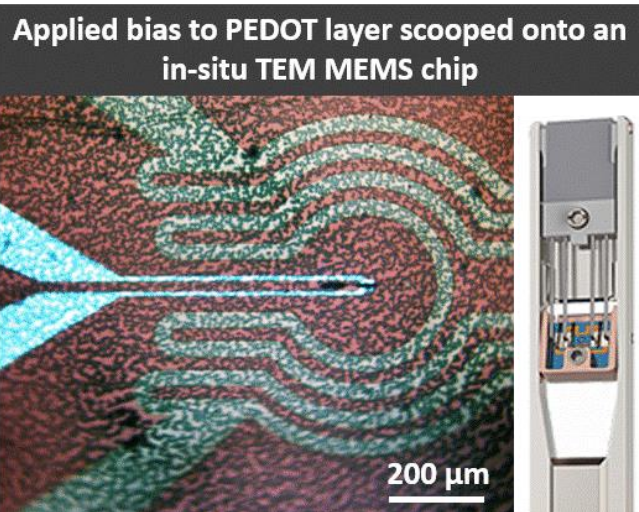

Figure 1. (a) Photograph of glass vial of PEDOT 2D layers suspended in acetone, showing the highly conducting surface, (b) low resolution BF and DF TEM imaging of the amorphous and fibrous nature of the PEDOT layers, (c) optical microscope image of the ex-situ biasing of PEDOT and (d) the PEDOT layer that was scooped onto the in-situ TEM DENS MEMS chips.

ov

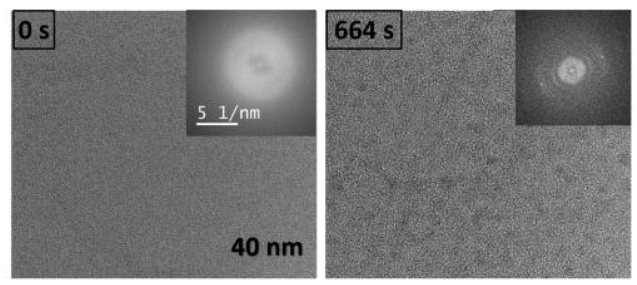

$3 \mathrm{~V}$

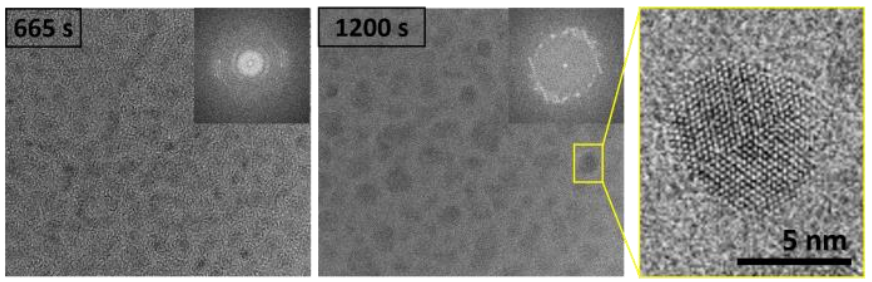

Figure 2. BF TEM imaging during in-situ biasing of phase change from amorphous to crystalline nanoparticles. 


\section{References}

[1] Lin, Zhaoyang, Y. H., Nature Electronics 2.9 (2019) 378-388.

[2] Caironi, M., \& Yong-Young N., Large area and flexible electronics. John Wiley \& Sons, 2015.

[3] Salvatore, G. A., et al. Nature communications 5.1 (2014) 1-8.

[4] Liu, Y., Matt P., \& Giovanni, A. S., ACS nano 11.10 (2017): 9614-9635.

[5] Ni, D., et al. Journal of Materials Chemistry A 7.3 (2019) 1323-1333.

[6] Fan, Xi, et al. Advanced Science 6.19 (2019): 1900813.

[7] Lu, B., et al. Nature communications 10.1 (2019): 1-10. 